\title{
Recursive representation of the torus 1-point conformal block
}

\author{
Leszek Hadasz ${ }^{\dagger 1}$ Zbigniew Jaskólski ${ }^{\ddagger 2}$ and Paulina Suchanek ${ }^{\ddagger 3}$ \\ $\dagger$ M. Smoluchowski Institute of Physics, Jagiellonian University \\ Reymonta 4, 30-059 Kraków, Poland, \\ $\ddagger$ Institute of Theoretical Physics, University of Wrocław \\ pl. M. Borna, 50-204 Wrocław, Poland.
}

\begin{abstract}
The recursive relation for the 1-point conformal block on a torus is derived and used to prove the identities between conformal blocks recently conjectured by Poghossian in [1]. As an illustration of the efficiency of the recurrence method the modular invariance of the 1-point Liouville correlation function is numerically analyzed.
\end{abstract}

PACS: 11.25.Hf, 11.30.Pb

\footnotetext{
${ }^{1}$ e-mail: hadasz@th.if.uj.edu.pl

${ }^{2} e$-mail: jask@ift.uni.wroc.pl

${ }^{3} e$-mail: paulina@ift.uni.wroc.pl
} 


\section{Introduction}

An interesting duality between certain class of $\mathrm{N}=2$ superconformal field theories in four dimensions and the two-dimensional Liouville field theory has been recently discovered by Alday, Gaiotto and Tachikawa [2]. An essential part of this relation actively studied in a number of papers [3-11] is an exact correspondence between instanton parts of Nekrasov partition functions in $\mathrm{N}=2 \mathrm{SCFT}$ and conformal blocks of the 2-dimensional CFT. This in particular concerns two basic objects of CFT: the 4-point conformal block on a sphere and the 1-point conformal block on a torus. These special cases has been recently analyzed by Poghossian [1] who applied the recursive relation for the 4-point blocks on a sphere discovered long time ago by Alexei Zamolodchikov in CFT [12-14] to the instanton part of the Nekrasov partition function with four fundamentals. He also proposed and verified to certain order a recursive relation for the Nekrasov function with one adjoint hypermultiplet. It was observed that by the AGT duality this yields previously unknown recursive relation for conformal blocks in 2-dimensional CFT.

The aim of the present paper is to provide a complete derivation of the recursive relation for 1-point conformal blocks on a torus conjectured by Poghossian [1]. Our method is based on the algebraic properties of the 3-point conformal blocks and the analytic structure of the inverse of the Gram matrices in Verma modules [15]. It parallels to large extend Zamolodchikov's original derivation for the 4-point blocks [12-14]. We set our notation in Section 2 and present the derivation in Section 3.

As an application of the recurrence relations we prove two important identities between conformal blocks conjectured by Poghossian [1]. The first one relates the 1-point block on a torus with certain 4-point elliptic blocks on a sphere. The second relates 4-point elliptic blocks for different values of central charges, external weights and the elliptic variable $q$. It had been motivated by the intriguing relation between the Liouville 1-point correlation function on the torus and the Liouville 4-point correlation function on the sphere recently proposed by Fateev, Litvinov, Neveu and Onofri [16]. The derivation of both relations is presented in Section 4. They in particular imply ${ }^{4}$ :

$$
\mathcal{H}_{c, \Delta_{\alpha}}^{\lambda}\left(q^{2}\right)=\mathcal{H}_{c^{\prime}, \Delta_{\alpha^{\prime}}^{\prime}}\left[\begin{array}{cc}
\frac{1}{2 b^{\prime}} & \frac{\lambda}{\sqrt{2}} \\
\frac{1}{2 b^{\prime}} & \frac{1}{2 b^{\prime}}
\end{array}\right](q), \quad b^{\prime}=\frac{b}{\sqrt{2}}, \quad \alpha^{\prime}=\sqrt{2} \alpha,
$$

and

$$
\mathcal{H}_{c, \Delta_{\alpha}}^{\lambda}\left(q^{2}\right)=\mathcal{H}_{c^{\prime}, \Delta_{\alpha^{\prime}}^{\prime}}\left[\begin{array}{cc}
\frac{b^{\prime}}{2} & \frac{\lambda}{\sqrt{2}} \\
\frac{b^{\prime}}{2} & \frac{b^{\prime}}{2}
\end{array}\right](q), \quad b^{\prime}=\sqrt{2} b, \quad \alpha^{\prime}=\sqrt{2} \alpha .
$$

\footnotetext{
${ }^{4}$ See the next section for our notation conventions.
} 
These identities, which can be seen as chiral versions of the identity for the Liouville correlation functions proposed in [16], constitute the most interesting results of the present work. They provide for instance a new tool for analyzing virtually untouched problem of modular invariance of nontrivial torus 1-point functions in CFT. We hope to report on some of they consequences in the forthcoming paper.

Beside theoretical applications the recursive relation for 1-point block on a torus gives a very efficient numerical method of analyzing the modular bootstrap in CFT. As an illustration of this method we present in Section 5 some numerical checks of modular bootstrap in the Liouville theory.

\section{1-point functions}

Consider a primary field $\phi_{\lambda, \bar{\lambda}}$ with the conformal weights $\left(\Delta_{\lambda}, \Delta_{\bar{\lambda}}\right)$ for which the following parametrization is assumed:

$$
\Delta_{\lambda}=\frac{1}{4}\left(Q^{2}-\lambda^{2}\right), \quad c=1+6 Q^{2}, \quad Q=b+b^{-1} .
$$

In terms of the CFT on the complex plane the 1-point correlation function of $\phi_{\lambda, \bar{\lambda}}$ on a torus takes the form:

$$
\left\langle\phi_{\lambda, \bar{\lambda}}\right\rangle=\operatorname{Tr}\left(e^{-(\operatorname{Im} \tau) \hat{H}+i(\operatorname{Re} \tau) \hat{P}} \phi_{\lambda}(1,1)\right)=(q \bar{q})^{-\frac{c}{24}} \operatorname{Tr}\left(q^{L_{0}} \bar{q}^{\bar{L}_{0}} \phi_{\lambda}(1,1)\right)
$$

where $\tau$ is the torus modular parameter and

$$
\hat{H}=2 \pi\left(L_{0}+\bar{L}_{0}\right)-\frac{\pi c}{6}, \quad \hat{P}=2 \pi\left(L_{0}-\bar{L}_{0}\right), \quad q=e^{2 \pi i \tau} .
$$

The trace can be calculated in the standard bases of Verma modules:

$$
\nu_{\Delta, M}=L_{-M} \nu_{\Delta} \equiv L_{-m_{j}} \ldots L_{-m_{1}} \nu_{\Delta}
$$

where $M=\left\{m_{1}, m_{2}, \ldots, m_{j}\right\} \subset \mathbb{N}$ stands for an arbitrary ordered set of indices $m_{j} \leq \ldots \leq$ $m_{2} \leq m_{1}$, and $\nu_{\Delta} \in \mathcal{V}_{\Delta}$ is the highest weight state. This yields:

$$
\begin{aligned}
\left\langle\phi_{\lambda, \bar{\lambda}}\right\rangle & =(q \bar{q})^{-\frac{c}{24}} \sum_{(\Delta, \bar{\Delta})} \sum_{n=0}^{\infty} q^{\Delta+n} \bar{q}^{\bar{\Delta}+n} \\
& \times \sum_{\substack{n=|M|=|N| \\
n=|\bar{M}|=|\bar{N}|}}\left[B_{c, \Delta}^{n}\right]^{M N}\left[\bar{B}_{c, \bar{\Delta}}^{n}\right]^{\bar{M} \bar{N}}\left\langle\nu_{\Delta, M} \otimes \bar{\nu}_{\bar{\Delta}, \bar{M}}\left|\phi_{\lambda, \bar{\lambda}}(1,1)\right| \nu_{\Delta, N} \otimes \bar{\nu}_{\bar{\Delta}, \bar{N}}\right\rangle
\end{aligned}
$$


where the sum over all weights $(\Delta, \bar{\Delta})$ from the spectrum of the theory is assumed, $|M|=$ $m_{1}+\ldots+m_{j}$ and $\left[B_{c, \Delta}^{n}\right]^{M N}$ is the inverse of the Gram matrix

$$
\left[B_{c, \Delta}^{n}\right]_{M N}=\left\langle\nu_{\Delta, N} \mid \nu_{\Delta, M}\right\rangle, \quad|M|=|N|=n
$$

at the level $n$.

The 3-point correlation function in (3) can be expressed as a product of the 3-point ("left" and "right") conformal blocks and a structure constant:

$$
\begin{gathered}
\left\langle\nu_{\Delta, M} \otimes \bar{\nu}_{\bar{\Delta}, \bar{M}}\left|\phi_{\lambda, \bar{\lambda}}(1,1)\right| \nu_{\Delta, N} \otimes \bar{\nu}_{\bar{\Delta}, \bar{N}}\right\rangle=\rho\left(\nu_{\Delta, N}, \nu_{\lambda}, \nu_{\Delta, M}\right) \rho\left(\nu_{\bar{\Delta}, \bar{N}}, \nu_{\bar{\lambda}}, \nu_{\bar{\Delta}, \bar{M}}\right) C_{\Delta, \bar{\Delta}}^{\lambda, \bar{\lambda}} \\
C_{\Delta, \bar{\Delta}}^{\lambda, \bar{\lambda}}=\left\langle\nu_{\Delta} \otimes \nu_{\bar{\Delta}}\left|\phi_{\lambda, \bar{\lambda}}(1,1)\right| \nu_{\Delta} \otimes \nu_{\bar{\Delta}}\right\rangle .
\end{gathered}
$$

Introducing the 1-point conformal block:

$$
\begin{aligned}
\mathcal{F}_{c, \Delta}^{\lambda}(q) & =q^{\Delta-\frac{c}{24}} \sum_{n=0}^{\infty} q^{n} F_{c, \Delta}^{\lambda, n}, \\
F_{c, \Delta}^{\lambda, n} & =\sum_{n=|M|=|N|} \rho\left(\nu_{\Delta, N}, \nu_{\lambda}, \nu_{\Delta, M}\right)\left[B_{c, \Delta}^{n}\right]^{M N},
\end{aligned}
$$

one can write the 1-point correlation function on the torus in the following form:

$$
\left\langle\phi_{\lambda, \bar{\lambda}}\right\rangle=\sum_{(\Delta, \bar{\Delta})} \mathcal{F}_{c, \Delta}^{\lambda}(q) \mathcal{F}_{c, \bar{\Delta}}^{\lambda}(\bar{q}) C_{\Delta, \bar{\Delta}}^{\lambda, \bar{\lambda}} .
$$

\section{Recursive relations}

For arbitrary vectors $\xi_{i} \in \mathcal{V}_{\Delta_{i}}$ the 3 -point block $\rho\left(\xi_{3}, \xi_{2}, \xi_{1}\right)$ is a polynomial function of the weights $\Delta_{i}$, completely determined by the conformal Ward identities [15] and the normalization condition $\rho\left(\nu_{3}, \nu_{2}, \nu_{1}\right)=1$. It follows from (4) that the block coefficients $F_{c, \Delta}^{\lambda, n}$ are polynomials in the external weight $\Delta_{\lambda}$ and rational functions of the central charge $c$ and the intermediate weight $\Delta$ with the locations of poles determined by the zeroes of the determinant of the Gram matrix $\left[B_{c, \Delta}^{n}\right]_{M, N}$.

In the generic case the Verma module $\mathcal{V}_{\Delta_{r s}+r s}$ is not reducible and the only singularities of $\left[B_{c, \Delta}^{n}\right]^{M, N}$ as a function of $\Delta$ are simple poles at

$$
\Delta_{r s}(c)=\frac{Q^{2}}{4}-\frac{1}{4}\left(r b+\frac{s}{b}\right)^{2}
$$

where $r, s \in \mathbb{Z}, \quad r \geq 1, s \geq 1, \quad 1 \leq r s \leq n$. 
As a function of $c$ the inverse Gram matrix at the level $n$ has simple poles at the locations

$$
\begin{aligned}
& c_{r s}(\Delta)=1+6\left(b_{r s}(\Delta)+\frac{1}{b_{r s}(\Delta)}\right)^{2} \\
& b_{r s}^{2}(\Delta)=\frac{1}{1-r^{2}}\left(r s-1+2 \Delta+\sqrt{(r-s)^{2}+4(r s-1) \Delta+4 \Delta^{2}}\right) .
\end{aligned}
$$

where $r, s \in \mathbb{Z}, \quad r \geq 2, s \geq 1, \quad 1 \leq r s \leq n$.

The block's coefficient $F_{c, \Delta}^{\lambda, n}$ can be expressed either as a sum over the poles in the intermediate weight:

$$
F_{c, \Delta}^{\lambda, n}=\mathrm{h}_{c}^{\lambda, n}+\sum_{1 \leq r s \leq n} \frac{\mathcal{R}_{c, r s}^{\lambda, n}}{\Delta-\Delta_{r s}(c)}
$$

or in the central charge:

$$
F_{c, \Delta}^{\lambda, n}=\mathrm{f}_{\Delta}^{\lambda, n}+\sum_{1<r s \leq n} \frac{\widetilde{\mathcal{R}}_{\Delta, r s}^{\lambda, n}}{c-c_{r s}(\Delta)}
$$

The residues at $\Delta_{r s}(c)$ and $c_{r s}(\Delta)$ are simply related:

$$
\begin{aligned}
\widetilde{\mathcal{R}}_{\Delta, r s}^{\lambda, n} & =-\frac{\partial c_{r s}(\Delta)}{\partial \Delta} \mathcal{R}_{c_{r s}(\Delta), r s}^{\lambda, n} \\
\frac{\partial c_{r s}(\Delta)}{\partial \Delta} & =4 \frac{c_{r s}(\Delta)-1}{\left(r^{2}-1\right)\left(b_{r s}(\Delta)\right)^{4}-\left(s^{2}-1\right)}
\end{aligned}
$$

In order to calculate the residue at $\Delta=\Delta_{r s}$ it is useful to choose a specific basis in the Verma module $\mathcal{V}_{\Delta}$. Let us introduce the state:

$$
\chi_{r s}^{\Delta}=\sum_{|M|=r s} \chi_{r s}^{M} L_{-M} \nu_{\Delta}
$$

where $\chi_{r s}^{M}$ are the coefficients of the singular vector $\chi_{r s}$ in the standard basis of $\mathcal{V}_{\Delta_{r s}}$ :

$$
\chi_{r s}=\sum_{|M|=r s} \chi_{r s}^{M} L_{-M} \nu_{\Delta_{r s}} .
$$

The family of states $\left\{L_{-N} \chi_{r s}^{\Delta}\right\}_{|N|=n-r s}$ can be completed to a full basis in the Verma module $\mathcal{V}_{\Delta}$ at the level $n>r s$. Working in this basis one gets [15]:

$$
\mathcal{R}_{c, r s}^{\lambda, n}=\lim _{\Delta \rightarrow \Delta_{r s}}\left(\Delta-\Delta_{r s}(c)\right) F_{c, \Delta}^{\lambda, n}=A_{r s}(c) \sum_{n-r s=|M|=|N|} \rho\left(L_{-N} \chi_{r s}, \nu_{\lambda}, L_{-M} \chi_{r s}\right)\left[B_{c, \Delta_{r s}+r s}^{n-r s}\right]^{M N}
$$

where

$$
A_{r s}(c)=\lim _{\Delta \rightarrow \Delta_{r s}}\left(\frac{\left\langle\chi_{r s}^{\Delta} \mid \chi_{r s}^{\Delta}\right\rangle}{\Delta-\Delta_{r s}(c)}\right)^{-1}
$$


It is convenient to normalize the singular vector $\chi_{r s}$ such that the coefficient in front of $\left(L_{-1}\right)^{r s}$ equals 1. For this normalization the exact form of the coefficient $A_{r s}(c)$ was first proposed by Al. Zamolodchikov in [12] and then justified in [17]. It reads:

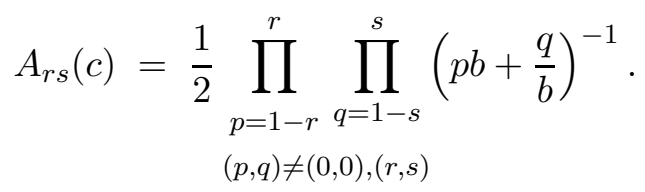

Using the factorization formula:

$$
\rho\left(L_{-N} \chi_{r s}, \nu_{\lambda}, L_{-M} \chi_{r s}\right)=\rho\left(L_{-N} \nu_{\Delta_{r s}+r s}, \nu_{\lambda}, L_{-M} \nu_{\Delta_{r s}+r s}\right) \rho\left(\chi_{r s}, \nu_{\lambda}, \chi_{r s}\right)
$$

one can show that the residue is proportional to the lower order block coefficient:

$$
\mathcal{R}_{c, r s}^{\lambda, n}=A_{r s}(c) \rho\left(\chi_{r s}, \nu_{\lambda}, \chi_{r s}\right) F_{c, \Delta_{r s}+r s}^{\lambda, n-r s}
$$

For the normalized singular vector $\chi_{r s}$ one gets [15]:

$$
\rho\left(\chi_{r s}, \nu_{\lambda}, \chi_{r s}\right)=\rho\left(\chi_{r s}, \nu_{\lambda}, \nu_{\Delta_{r s}+r s}\right) \rho\left(\nu_{\Delta_{r s}}, \nu_{\lambda}, \chi_{r s}\right)=P_{c}^{r s}\left[\begin{array}{c}
\Delta_{\lambda} \\
\Delta_{r s}+r s
\end{array}\right] P_{c}^{r s}\left[\begin{array}{c}
\Delta_{\lambda} \\
\Delta_{r s}
\end{array}\right]
$$

where the fusion polynomials are defined by:

$$
P_{c}^{r s}\left[\begin{array}{c}
\Delta_{2} \\
\Delta_{1}
\end{array}\right]=\prod_{\substack{p=1-r \\
p+r=1 \bmod 2}}^{r-1} \prod_{\substack{q=1-s \\
q+s=1 \bmod 2}}^{s-1}\left(\frac{\lambda_{2}+\lambda_{1}+p b+q b^{-1}}{2}\right)\left(\frac{\lambda_{2}-\lambda_{1}+p b+q b^{-1}}{2}\right)
$$

and $\Delta_{i}=\frac{1}{4}\left(Q^{2}-\lambda_{i}^{2}\right)$. In the case under consideration:

$$
\begin{aligned}
P_{c}^{r s}\left[\begin{array}{c}
\Delta_{\lambda} \\
\Delta_{r s}+r s
\end{array}\right] P_{c}^{r s}\left[\begin{array}{c}
\Delta_{\lambda} \\
\Delta_{r s}
\end{array}\right] & =\prod_{\substack{k=1 \\
k=1 \bmod 2}}^{2 r-1} \prod_{l=1}^{2 s-1}\left(\frac{\lambda+k b+l b^{-1}}{2}\right) \\
& \times\left(\frac{\lambda-k b-l b^{-1}}{2}\right)\left(\frac{\lambda+k b-l b^{-1}}{2}\right)\left(\frac{\lambda-k b+l b^{-1}}{2}\right) .
\end{aligned}
$$

The last step in our derivation is to find the non-singular terms in the equations (5), (6). Let us start with the expansion (6). Since $\mathrm{f}_{\Delta}^{\lambda, n}$ does not depend on the central charge it can be calculated from the $c \rightarrow \infty$ limit:

$$
\sum_{n=0}^{\infty} q^{n} \mathrm{f}_{\Delta}^{\lambda, n}=\lim _{c \rightarrow \infty} q^{-\Delta+\frac{c}{24}} \mathcal{F}_{c, \Delta}^{\lambda}(q) .
$$

Note that the block's coefficients (4) depend on $c$ only via the inverse Gram matrix $\left[B_{c, \Delta}^{n}\right]^{M, N}$. Analyzing the polynomial dependence of the Gram matrix minors on $c$ one can show [15] that 
the only element of the inverse Gram matrix which does not vanish in the limit $c \rightarrow \infty$ is the diagonal one corresponding to the state $L_{-1}^{n}\left|\nu_{\Delta}\right\rangle$ :

$$
\lim _{c \rightarrow \infty}\left[B_{c, \Delta}^{n}\right]^{\mathbb{I} \mathbb{I}}=\frac{1}{\left\langle\nu_{\Delta}\left|L_{1}^{n} L_{-1}^{n}\right| \nu_{\Delta}\right\rangle}=\frac{1}{n !(2 \Delta)_{n}},
$$

where $(a)_{n}=\frac{\Gamma(a+n)}{\Gamma(a)}$ is the Pochhammer symbol. The 3-point block for this state is given by:

$$
\rho\left(L_{-1}^{n} \nu_{\Delta}, \nu_{\lambda}, L_{-1}^{n} \nu_{\Delta}\right)=\sum_{k=0}^{n} \frac{1}{(n-k) !}\left(\frac{n !}{k !}\right)^{2} \frac{\Gamma(2 \Delta+n)}{\Gamma(2 \Delta+k)} \frac{\Gamma\left(\Delta_{\lambda}+k\right)}{\Gamma\left(\Delta_{\lambda}-k\right)} .
$$

Thus the $c \rightarrow \infty$ limit of the 1 -point block reads:

$$
\lim _{c \rightarrow \infty} q^{-\Delta+\frac{c}{24}} \mathcal{F}_{c, \Delta}^{\lambda}(q)=\sum_{n=0}^{\infty} q^{n} \sum_{k=0}^{n} \frac{n !}{(n-k) !(k !)^{2}} \frac{\Gamma(2 \Delta)}{\Gamma(2 \Delta+k)} \frac{\Gamma\left(\Delta_{\lambda}+k\right)}{\Gamma\left(\Delta_{\lambda}-k\right)},
$$

which yields:

$$
\sum_{n=0}^{\infty} q^{n} \mathrm{f}_{\Delta}^{\lambda, n}=\frac{1}{1-q}{ }_{2} F_{1}\left(\Delta_{\lambda}, 1-\Delta_{\lambda} ; 2 \Delta ; \frac{q}{q-1}\right) .
$$

The $\Delta \rightarrow \infty$ asymptotics of the block is even easier to obtain and leads to a more convenient recursion. With the help of the Ward identities one easily shows that for $\Delta \rightarrow \infty$ :

$$
\rho\left(\nu_{\Delta, N}, \nu_{\lambda}, \nu_{\Delta, M}\right)=\rho\left(\nu_{\Delta, N}, \nu_{Q}, \nu_{\Delta, M}\right)\left(1+\mathcal{O}\left(\Delta^{-1}\right)\right)
$$

where $\nu_{Q}$ is the highest weight state in the vacuum Verma module $\left(\Delta_{Q}=0\right)$. Since (for $|M|=|N|=n)$ :

$$
\rho\left(\nu_{\Delta, N}, \nu_{Q}, \nu_{\Delta, M}\right)=\left[B_{c, \Delta}^{n}\right]_{N M}
$$

we get:

$$
\mathrm{h}_{c}^{\lambda, n}=\lim _{\Delta \rightarrow \infty} F_{c, \Delta}^{\lambda, n}=\sum_{|M|=|N|=n}\left[B_{c, \Delta}^{n}\right]_{N M}\left[B_{c, \Delta}^{n}\right]^{M N}=\sum_{|N|=n} \delta_{N}^{N}=p(n)
$$

where $p(n)$ denotes the number of ways $n$ can be written as a sum of positive integers. Thus

$$
\lim _{\Delta \rightarrow \infty}\left(q^{\frac{c}{24}-\Delta} \mathcal{F}_{c, \Delta}^{\lambda}(q)\right)=\prod_{n=1}^{\infty}\left(1-q^{n}\right)^{-1} .
$$

This suggest the following definition of the elliptic 1-point block on a torus:

$$
\mathcal{H}_{c, \Delta}^{\lambda}(q)=q^{\frac{c-1}{24}-\Delta} \eta(q) \mathcal{F}_{c, \Delta}^{\lambda}(q)=\sum_{n=0}^{\infty} q^{n} H_{c, \Delta}^{\lambda, n},
$$

where $\eta(q)$ is the Dedekind eta function. One easily checks that the coefficients $H_{c, \Delta}^{\lambda, n}$ have essentially the same pole structure as the coefficients $F_{c, \Delta}^{\lambda, n}$ and the following recursive formula holds:

$$
H_{c, \Delta}^{\lambda, n}=\delta_{0}^{n}+\sum_{1 \leq r s \leq n} \frac{A_{r s}(c) P_{c}^{r s}\left[\begin{array}{c}
\Delta_{\lambda} \\
\Delta_{r s}+r s
\end{array}\right] P_{c}^{r s}\left[\begin{array}{c}
\Delta_{\lambda} \\
\Delta_{r s}
\end{array}\right]}{\Delta-\Delta_{r s}(c)} H_{c, \Delta_{r s}+r s}^{\lambda, n-r s}
$$


Let us note that the form of the regular terms in (9) can be also deduced from the limiting case $\Delta \rightarrow 0(\lambda \rightarrow Q)$ where the fusion polynomials, and therefore all the residua, vanish and the 1-point block become the Virasoro character.

\section{Poghossian identities}

Using (8) one can check the identity:

$$
\begin{aligned}
P_{c}^{r s}\left[\begin{array}{c}
\Delta_{\lambda} \\
\Delta_{r s}+r s
\end{array}\right] P_{c}^{r s}\left[\begin{array}{c}
\Delta_{\lambda} \\
\Delta_{r s}
\end{array}\right]=\prod_{\substack{p=1-r \\
p+r=1 \bmod 2}}^{r-1} \prod_{\substack{q=1-s \\
q+s=1 \bmod 2}}^{s-1}\left(\frac{\lambda}{2}+p b+q b^{-1}+\frac{Q}{2}\right) \\
\times\left(\frac{\lambda}{2}+p b+q b^{-1}-\frac{Q}{2}\right)\left(\frac{\lambda}{2}+p b+q b^{-1}+\frac{b}{2}-\frac{1}{2 b}\right)\left(\frac{\lambda}{2}+p b+q b^{-1}-\frac{b}{2}+\frac{1}{2 b}\right) .
\end{aligned}
$$

The r.h.s. can be identified as a product of fusion polynomials:

$$
P_{c}^{r s}\left[\begin{array}{c}
\Delta_{\lambda} \\
\Delta_{r s}+r s
\end{array}\right] P_{c}^{r s}\left[\begin{array}{c}
\Delta_{\lambda} \\
\Delta_{r s}
\end{array}\right]=(16)^{r s} P_{c}^{r s}\left[\begin{array}{c}
\Delta_{2} \\
\Delta_{1}
\end{array}\right] P_{c}^{r s}\left[\begin{array}{c}
\Delta_{3} \\
\Delta_{4}
\end{array}\right]
$$

provided that one of the equalities:

$$
\begin{aligned}
\left(\lambda_{1}, \lambda_{2}, \lambda_{3}, \lambda_{4}\right) & =\left(\frac{Q}{2}, \frac{\lambda}{2}, \frac{\lambda}{2}, \frac{b}{2}-\frac{1}{2 b}\right), \\
\left(\lambda_{1}, \lambda_{2}, \lambda_{3}, \lambda_{4}\right) & =\left(\frac{1}{2 b}, \frac{\lambda}{2}+\frac{b}{2}, \frac{\lambda}{2}-\frac{b}{2}, \frac{1}{2 b}\right), \\
\left(\lambda_{1}, \lambda_{2}, \lambda_{3}, \lambda_{4}\right) & =\left(\frac{b}{2}, \frac{\lambda}{2}+\frac{1}{2 b}, \frac{\lambda}{2}-\frac{1}{2 b}, \frac{b}{2}\right),
\end{aligned}
$$

hold. Let us now recall that in the case of the 4-point elliptic block on the sphere

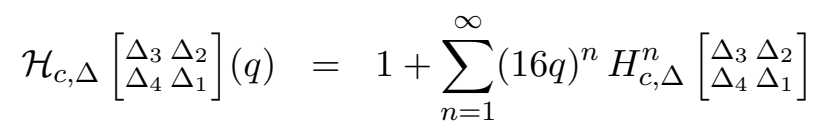

the recursive relation takes the form $[13,14]$ :

$$
H_{c, \Delta}^{n}\left[\begin{array}{l}
\Delta_{3} \Delta_{2} \\
\Delta_{4} \Delta_{1}
\end{array}\right]=\delta_{0}^{n}+\sum_{1 \leq r s \leq n} \frac{A_{r s}(c) P_{c}^{r s}\left[\begin{array}{c}
\Delta_{2} \\
\Delta_{1}
\end{array}\right] P_{c}^{r s}\left[\begin{array}{c}
\Delta_{3} \\
\Delta_{4}
\end{array}\right]}{\Delta-\Delta_{r s}(c)} H_{c, \Delta_{r s}+r s}^{n-r s}\left[\begin{array}{ll}
\Delta_{3} & \Delta_{2} \\
\Delta_{4} & \Delta_{1}
\end{array}\right] .
$$

Since the solutions to the recursive formulae (9) and (12) are unique we get by comparing (9), (10) and (12) that

$$
H_{c, \Delta}^{\lambda, n}=16^{n} H_{c, \Delta}^{n}\left[\begin{array}{ll}
\Delta_{3} & \Delta_{2} \\
\Delta_{4} & \Delta_{1}
\end{array}\right]
$$

and therefore

$$
\mathcal{H}_{c, \Delta}^{\lambda}(q)=\mathcal{H}_{c, \Delta}\left[\begin{array}{cc}
\frac{1}{2} \lambda & \frac{1}{2} \lambda \\
\frac{1}{2}\left(b-\frac{1}{b}\right) & \frac{1}{2}\left(b+\frac{1}{b}\right)
\end{array}\right](q)=\mathcal{H}_{c, \Delta}\left[\begin{array}{cc}
\frac{1}{2}(\lambda-b) & \frac{1}{2}(\lambda+b) \\
\frac{1}{2 b} & \frac{1}{2 b}
\end{array}\right](q)=\mathcal{H}_{c, \Delta}\left[\begin{array}{cc}
\frac{1}{2}\left(\lambda-\frac{1}{b}\right) & \frac{1}{2}\left(\lambda+\frac{1}{b}\right) \\
\frac{1}{2} b & \frac{1}{2} b
\end{array}\right](q) .
$$


We have thus obtained a simple proof of the first relation proposed in [1].

In the present notation the second relation conjectured in [1] reads:

$$
\begin{aligned}
\mathcal{H}_{c, \Delta}\left[\begin{array}{cc}
\frac{1}{2 b} & \mu \\
\frac{1}{2 b} & \frac{1}{2 b}
\end{array}\right](q) & =\mathcal{H}_{c^{\prime}, \Delta^{\prime}}\left[\begin{array}{cc}
\frac{1}{\sqrt{2}} \mu & \frac{1}{\sqrt{2}} \mu \\
\frac{1}{2}\left(b^{\prime}-\frac{1}{b^{\prime}}\right) & \frac{1}{2}\left(b^{\prime}+\frac{1}{b^{\prime}}\right)
\end{array}\right]\left(q^{2}\right) \\
& =\mathcal{H}_{c^{\prime}, \Delta^{\prime}}\left[\begin{array}{cc}
\frac{1}{\sqrt{2}} \mu-\frac{1}{2} b^{\prime} & \frac{1}{\sqrt{2}} \mu+\frac{1}{2} b^{\prime} \\
\frac{1}{2 b^{\prime}} & \frac{1}{2 b^{\prime}}
\end{array}\right]\left(q^{2}\right) \\
& =\mathcal{H}_{c^{\prime}, \Delta^{\prime}}\left[\begin{array}{cc}
\frac{1}{\sqrt{2}} \mu-\frac{1}{2 b^{\prime}} & \frac{1}{\sqrt{2}} \mu+\frac{1}{2 b^{\prime}} \\
\frac{1}{2} b^{\prime} & \frac{1}{2} b^{\prime}
\end{array}\right]\left(q^{2}\right) .
\end{aligned}
$$

where:

$$
\begin{aligned}
& c^{\prime}=1+6\left(b^{\prime}+\frac{1}{b^{\prime}}\right)^{2}, \quad \Delta_{\lambda^{\prime}}^{\prime}=\frac{\left(b^{\prime}+\frac{1}{b^{\prime}}\right)^{2}}{4}-\frac{\left(\lambda^{\prime}\right)^{2}}{4}, \\
& b^{\prime}=\sqrt{2} b, \quad \lambda^{\prime}=\frac{\lambda}{\sqrt{2}} .
\end{aligned}
$$

There is also another relation of a similar origin with $b^{\prime}=\frac{b}{\sqrt{2}}$ :

$$
\begin{aligned}
\mathcal{H}_{c, \Delta}\left[\begin{array}{cc}
\frac{b}{2} & \mu \\
\frac{b}{2} & \frac{b}{2}
\end{array}\right](q) & =\mathcal{H}_{c^{\prime}, \Delta^{\prime}}\left[\begin{array}{cc}
\frac{1}{\sqrt{2}} \mu & \frac{1}{\sqrt{2}} \mu \\
\frac{1}{2}\left(b^{\prime}-\frac{1}{b^{\prime}}\right) & \frac{1}{2}\left(b^{\prime}+\frac{1}{b^{\prime}}\right)
\end{array}\right]\left(q^{2}\right) \\
& =\mathcal{H}_{c^{\prime}, \Delta^{\prime}}\left[\begin{array}{cc}
\frac{1}{\sqrt{2}} \mu-\frac{1}{2} b^{\prime} & \frac{1}{\sqrt{2}} \mu+\frac{1}{2} b^{\prime} \\
\frac{1}{2 b^{\prime}} & \frac{1}{2 b^{\prime}}
\end{array}\right]\left(q^{2}\right) \\
& =\mathcal{H}_{c^{\prime}, \Delta^{\prime}}\left[\begin{array}{cc}
\frac{1}{\sqrt{2}} \mu-\frac{1}{2 b^{\prime}} & \frac{1}{\sqrt{2}} \mu+\frac{1}{2 b^{\prime}} \\
\frac{1}{2} b^{\prime} & \frac{1}{2} b^{\prime}
\end{array}\right]\left(q^{2}\right) .
\end{aligned}
$$

We shall show that relations of the form:

$$
\mathcal{H}_{c, \Delta}\left[\begin{array}{ll}
\eta & \mu \\
\eta & \eta
\end{array}\right](q)=\mathcal{H}_{c^{\prime}, \Delta^{\prime}}\left[\begin{array}{cc}
\lambda_{3}^{\prime} & \lambda_{2}^{\prime} \\
\lambda_{4}^{\prime} & \lambda_{1}^{\prime}
\end{array}\right]\left(q^{2}\right)
$$

are to large extent unique. Let us first observe that the residua of the coefficients of $\mathcal{H}_{c, \Delta}\left[\begin{array}{ll}\eta & \mu \\ \eta & \eta\end{array}\right](q)$ contain the fusion polynomial

$$
P_{c}^{r s}\left[\begin{array}{l}
\eta \\
\eta
\end{array}\right]=\prod_{\substack{p=1-r \\
p+r=1 \bmod 2}}^{r-1} \prod_{\substack{q=1-s \\
q+s=1 \bmod 2}}^{s-1}\left(\frac{2 \eta+p b+q b^{-1}}{2}\right)\left(\frac{p b+q b^{-1}}{2}\right)
$$

which always vanishes if both $r$ and $s$ are odd. Moreover if $\eta=\frac{1}{2 b}$ it vanishes for odd $r$ and all $s .{ }^{5}$ Since $H_{c, \Delta}^{1}\left[\begin{array}{l}\eta \\ \eta\end{array} \eta\right]=0$, it follows that for $\eta=\frac{1}{2 b}$ all the odd coefficients of $\mathcal{H}_{c, \Delta}\left[\begin{array}{l}\eta \\ \eta\end{array}\right](q)$ vanish and the even ones satisfy the recursive relation:

$$
H_{c, \Delta}^{2 m}\left[\begin{array}{ll}
\eta & \mu \\
\eta & \eta
\end{array}\right]=\delta_{0}^{n}+\sum_{\substack{k, s \in \mathbb{N} \\
1 \leq k s \leq m}} \frac{R_{c}^{2 k, s}\left[\begin{array}{ll}
\eta & \mu \\
\eta & \eta
\end{array}\right]}{\Delta-\Delta_{2 k, s}} H_{c, \Delta_{2 k, s}+2 k s}^{2 m-2 k s}\left[\begin{array}{l}
\eta \\
\eta \\
\eta
\end{array}\right] .
$$

\footnotetext{
${ }^{5}$ The case $\eta=\frac{b}{2}$ leading to the relation (16) can be analyzed in a similar way.
} 
This is to be compared with the recursive relation:

$$
H_{b^{\prime}, \Delta^{\prime}}^{m}\left[\begin{array}{cc}
\lambda_{3}^{\prime} & \lambda_{2}^{\prime} \\
\lambda_{4}^{\prime} & \lambda_{1}^{\prime}
\end{array}\right]=\delta_{0}^{n}+\sum_{\substack{k, s \in \mathbb{N} \\
1 \leq k s \leq m}} \frac{R_{c^{\prime}}^{k, s}\left[\begin{array}{cc}
\lambda_{3}^{\prime} & \lambda_{2}^{\prime} \\
\lambda_{4}^{\prime} & \lambda_{1}^{\prime}
\end{array}\right]}{\Delta^{\prime}-\Delta_{r s}^{\prime}} H_{b^{\prime}, \Delta_{k, s}^{\prime}+k s}^{m-k s}\left[\begin{array}{cc}
\lambda_{3}^{\prime} & \lambda_{2}^{\prime} \\
\lambda_{4}^{\prime} & \lambda_{1}^{\prime}
\end{array}\right] .
$$

The numbers of terms in both relations coincide. Moreover for the identification of parameters (15):

$$
\left(\Delta-\Delta_{2 k, s}\right)=2\left[\frac{1}{4}\left(\sqrt{2} k b+\frac{s}{\sqrt{2} b}\right)^{2}-\frac{\lambda^{2}}{8}\right]=2\left(\Delta^{\prime}-\Delta_{k, s}^{\prime}\right),
$$

and:

$$
\Delta_{2 k s}+2 k s=\Delta_{k s}^{\prime}+k s
$$

The fusion polynomials can be expressed in the form:

$$
\begin{aligned}
& P_{c}^{2 k, s}\left[\begin{array}{c}
\frac{1}{2 b} \\
\frac{1}{2 b}
\end{array}\right] P_{c}^{2 k, s}\left[\begin{array}{c}
\mu \\
\frac{1}{2 b}
\end{array}\right]=4^{-k s} \prod_{\substack{p=1-2 k \\
p+2 k=1 \bmod 2}}^{2 k-1} \prod_{\substack{q=1-s \\
q+s=1 \bmod 2}}^{s-1}\left(\frac{p b+(q+1) b^{-1}}{2}\right)\left(\frac{p b+q b^{-1}}{2}\right) \\
& \times \prod_{\substack{p=1-k r \\
p+k=1 \bmod 2}} \prod_{\substack{q=1-s \\
q+s=1 \bmod 2}}^{s-1}\left(\left(\frac{\mu}{\sqrt{2}}+\frac{1}{2 b^{\prime}}-\frac{b^{\prime}}{2}+p b^{\prime}+\frac{q}{b^{\prime}}\right)\left(\frac{\mu}{\sqrt{2}}-\frac{1}{2 b^{\prime}}-\frac{b^{\prime}}{2}+p b^{\prime}+\frac{q}{b^{\prime}}\right)\right. \\
&\left.\quad\left(\frac{\mu}{\sqrt{2}}+\frac{1}{2 b^{\prime}}+\frac{b^{\prime}}{2}+p b^{\prime}+\frac{q}{b^{\prime}}\right)\left(\frac{\mu}{\sqrt{2}}-\frac{1}{2 b^{\prime}}+\frac{b^{\prime}}{2}+p b^{\prime}+\frac{q}{b^{\prime}}\right)\right) .
\end{aligned}
$$

As in the case of our previous derivation the last two lines can be identified as the fusion polynomials:

$$
\begin{aligned}
P_{c}^{2 k, s}\left[\begin{array}{c}
\frac{1}{2 b} \\
\frac{1}{2 b}
\end{array}\right] P_{c}^{2 k, s}\left[\begin{array}{c}
\mu \\
\frac{1}{2 b}
\end{array}\right] & =4^{-k s} \prod_{\substack{p=1-2 k \\
p+2 k=1 \bmod 2}}^{2 k-1} \prod_{\substack{q=1-s \\
q+s=1 \bmod 2}}^{s-1}\left(\frac{p b+(q+1) b^{-1}}{2}\right)\left(\frac{p b+q b^{-1}}{2}\right) \\
& \times(16)^{k s} P_{c^{\prime}}^{k s}\left[\begin{array}{c}
\lambda_{2}^{\prime} \\
\lambda_{1}^{\prime}
\end{array}\right] P_{c^{\prime}}^{k s}\left[\begin{array}{c}
\lambda_{3}^{\prime} \\
\lambda_{4}^{\prime}
\end{array}\right]
\end{aligned}
$$

where one of the following choices is assumed:

$$
\begin{aligned}
\left(\lambda_{1}^{\prime}, \lambda_{2}^{\prime}, \lambda_{3}^{\prime}, \lambda_{4}^{\prime}\right) & =\left(\frac{\mu}{\sqrt{2}}, \frac{b^{\prime}}{2}+\frac{1}{2 b^{\prime}}, \frac{\mu}{\sqrt{2}}, \frac{b^{\prime}}{2}-\frac{1}{2 b^{\prime}}\right), \\
\left(\lambda_{1}^{\prime}, \lambda_{2}^{\prime}, \lambda_{3}^{\prime}, \lambda_{4}^{\prime}\right) & =\left(\frac{\mu}{\sqrt{2}}+\frac{b^{\prime}}{2}, \frac{1}{2 b^{\prime}}, \frac{\mu}{\sqrt{2}}-\frac{b^{\prime}}{2}, \frac{1}{2 b^{\prime}}\right), \\
\left(\lambda_{1}^{\prime}, \lambda_{2}^{\prime}, \lambda_{3}^{\prime}, \lambda_{4}^{\prime}\right) & =\left(\frac{\mu}{\sqrt{2}}+\frac{1}{2 b^{\prime}}, \frac{b^{\prime}}{2}, \frac{\mu}{\sqrt{2}}-\frac{1}{2 b^{\prime}}, \frac{b^{\prime}}{2}\right) .
\end{aligned}
$$


Finally calculating the coefficients $A_{r s}$ one gets:

$$
A_{2 k, s}(c)=2^{1-2 k s} A_{k s}\left(c^{\prime}\right) \prod_{\substack{p=1-2 k \\ p+2 k=1 \bmod 2}}^{2 k-1} \prod_{\substack{q=1-s \\ q+s=1 \bmod 2}}^{s-1}\left(p b+(q+1) b^{-1}\right)^{-1}\left(p b+q b^{-1}\right)^{-1}
$$

where:

$$
\begin{aligned}
& A_{k s}\left(c^{\prime}\right)=\frac{1}{2} \prod_{p=1-k}^{k} \prod_{q=1-s}^{s}\left(p b^{\prime}+\frac{q}{b^{\prime}}\right)^{-1} \\
& (p, q) \neq(0,0),(k, s)
\end{aligned}
$$

Taking into account formulae (18), (19), (20) and (22) one obtains:

$$
\frac{R_{c}^{2 k, s}\left[\begin{array}{cc}
\frac{1}{2 b} & \mu \\
\frac{1}{2 b} & \frac{1}{2 b}
\end{array}\right]}{\Delta-\Delta_{r s}}=16^{-k s} \frac{R_{c^{\prime}}^{k, s}\left[\begin{array}{cc}
\lambda_{3}^{\prime} & \lambda_{2}^{\prime} \\
\lambda_{4}^{\prime} & \lambda_{1}^{\prime}
\end{array}\right]}{\left(\Delta^{\prime}-\Delta_{r s}^{\prime}\right)} .
$$

Hence the coefficients $H_{c, \Delta}^{2 m}\left[\begin{array}{l}\eta \\ \eta\end{array}\right]$ and $(16)^{-m} H_{b^{\prime}, \Delta^{\prime}}^{m}\left[\begin{array}{ll}\lambda_{3}^{\prime} & \lambda_{2}^{\prime} \\ \lambda_{4}^{\prime} & \lambda_{1}^{\prime}\end{array}\right]$ with the weights $(21)$ satisfy the same recursive relations. This completes our proof of the formula (14). Formula (16) can be derived along the same lines starting with $\eta=\frac{b}{2}$.

\section{Modular bootstrap in Liouville theory}

The modular invariance of the 1-point function on the torus:

$$
\left\langle\phi_{\lambda, \bar{\lambda}}\right\rangle_{-\frac{1}{\tau}}=(-1)^{\Delta_{\lambda}-\Delta_{\bar{\lambda}}} \tau^{\Delta_{\lambda}} \bar{\tau}^{\Delta_{\bar{\lambda}}}\left\langle\phi_{\lambda, \bar{\lambda}}\right\rangle_{\tau}
$$

along with the crossing invariance of the 4-point function on the sphere form the basic consistency conditions for any CFT on closed surfaces [18]. In the case of the Liouville theory the 1-point function can be expressed in terms of the elliptic blocks as follows:

$$
\left\langle\phi_{\lambda}\right\rangle_{\tau}=\int_{i \mathbb{R}_{+}} \frac{\mathrm{d} \alpha}{2 i}\left|q^{\Delta_{\alpha}-\frac{Q^{2}}{4}} \eta(q)^{-1} \mathcal{H}_{c, \Delta_{\alpha}}^{\lambda}(q)\right|^{2} C_{\Delta_{\alpha}}^{\lambda}
$$

where $q=\mathrm{e}^{2 \pi i \tau}$. Condition (23) then takes the form:

$$
\int_{i \mathbb{R}_{+}} \frac{\mathrm{d} \alpha}{2 i}\left|\tilde{q}^{-\frac{\alpha^{2}}{4}} \mathcal{H}_{c, \Delta_{\alpha}}^{\lambda}(\tilde{q})\right|^{2} C_{\Delta_{\alpha}}^{\lambda}=|\tau|^{2 \Delta_{\lambda}+1} \int_{i \mathbb{R}} \frac{\mathrm{d} \alpha}{2 i}\left|q^{-\frac{\alpha^{2}}{4}} \mathcal{H}_{c, \Delta_{\alpha}}^{\lambda}(q)\right|^{2} C_{\Delta_{\alpha}}^{\lambda}
$$

where and $\tilde{q}=\mathrm{e}^{-2 \pi i \frac{1}{\tau}}$. The Liouville structure constant reads [19]:

$$
C_{\Delta_{\alpha}}^{\lambda}=\left[\pi \mu \gamma\left(b^{2}\right) b^{2-2 b^{2}}\right]^{-\frac{Q+\lambda}{2 b}} \times \frac{\Upsilon_{0} \Upsilon(Q+\lambda) \Upsilon(\alpha) \Upsilon(-\alpha)}{\Upsilon^{2}\left(\frac{Q}{2}+\frac{\lambda}{2}\right) \Upsilon\left(\frac{Q}{2}+\frac{\lambda}{2}+\alpha\right) \Upsilon\left(\frac{Q}{2}+\frac{\lambda}{2}-\alpha\right)} .
$$


Separating the $\alpha$-dependent part

$$
\begin{aligned}
& C_{\Delta_{\alpha}}^{\lambda}=4\left[\pi \mu \gamma\left(b^{2}\right) b^{2-2 b^{2}}\right]^{-\frac{Q+\lambda}{2 b}} \times \frac{\Upsilon_{0} \Upsilon(Q+\lambda)}{\Upsilon^{2}\left(\frac{Q}{2}+\frac{\lambda}{2}\right)} r(\alpha) \\
& r(\alpha)=-\frac{\alpha^{2}}{4} \exp \int_{0}^{\infty} \frac{\mathrm{d} t}{t}\left(\frac{1+b^{4}-b^{2}\left(\lambda^{2}+2\right)}{2 b^{2}} e^{-t}+\cosh (\alpha t) \frac{\cosh \left(\frac{t \lambda}{2}\right)-\cosh \left(\frac{\left(b^{2}-1\right) t}{2 b}\right)}{\sinh \left(\frac{b t}{2}\right) \sinh \left(\frac{t}{2 b}\right)}\right)
\end{aligned}
$$

one can write $(24)$ as:

$$
\int_{i \mathbb{R}} \frac{\mathrm{d} \alpha}{2 i}\left|\tilde{q}^{-\frac{\alpha^{2}}{4}} \mathcal{H}_{c, \Delta_{\alpha}}^{\lambda}(\tilde{q})\right|^{2} r(\alpha)=|\tau|^{2 \Delta_{\lambda}+1} \int_{i \mathbb{R}} \frac{\mathrm{d} \alpha}{2 i}\left|q^{-\frac{\alpha^{2}}{4}} \mathcal{H}_{c, \Delta_{\alpha}}^{\lambda}(q)\right|^{2} r(\alpha) .
$$

This relation can be numerically analyzed with the help of the recursion relations derived in Section 3. Due to the rapidly oscillating integrand in (25) the numerical calculation of function $r(t)$ has to be carefully done. We present a sample of the calculations for $c=2, \lambda=i$ and for the modular parameter $\tau$ along the imaginary axis in the range $[0.2 i, 5 i]$. The results for the elliptic block expanded up to the term $q^{n}, n=4,5,6$ are presented on Fig.1. where the relative difference of the left and the right side of (25) is plotted.

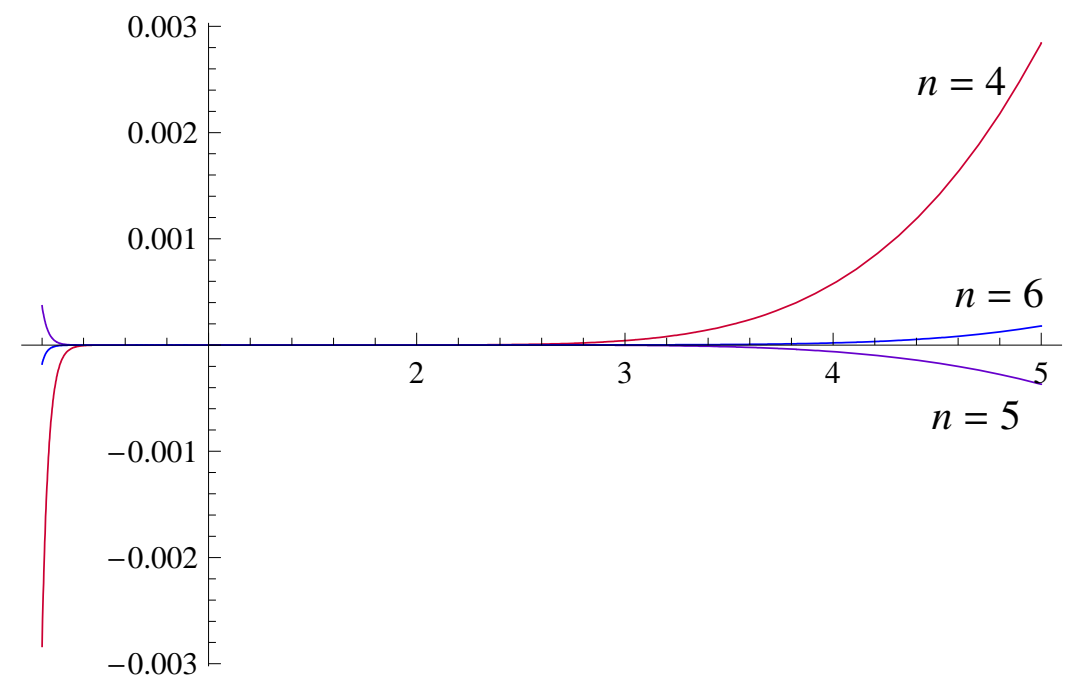

Fig.1 Numerical check of the modular bootstrap.

\section{Acknowledgements}

This work was supported by the Polish State Research Committee (KBN) grant no. N N202 0859 33. The work of L.H. was also supported by MNII grant 189/6.PRUE/2007/7. 


\section{References}

[1] R. Poghossian, Recursion relations in CFT and N=2 SYM theory, arXiv:0909.3412 [hepth].

[2] L. F. Alday, D. Gaiotto and Y. Tachikawa, Liouville Correlation Functions from Fourdimensional Gauge Theories, arXiv:0906.3219 [hep-th].

[3] N. Wyllard, $A_{N-1}$ conformal Toda field theory correlation functions from conformal $N=2 S U(N)$ quiver gauge theories, arXiv:0907.2189 [hep-th].

[4] A. Marshakov, A. Mironov and A. Morozov, On Combinatorial Expansions of Conformal Blocks, arXiv:0907.3946 [hep-th].

[5] D. Gaiotto, Asymptotically free $N=2$ theories and irregular conformal blocks, arXiv:0908.0307 [hep-th].

[6] A. Mironov, S. Mironov, A. Morozov and A. Morozov, CFT exercises for the needs of AGT, arXiv:0908.2064 [hep-th].

[7] L. F. Alday, D. Gaiotto, S. Gukov, Y. Tachikawa and H. Verlinde, Loop and surface operators in $N=2$ gauge theory and Liouville modular geometry, arXiv:0909.0945 [hepth].

[8] A. Marshakov, A. Mironov and A. Morozov, Zamolodchikov asymptotic formula and instanton expansion in $N=2 S U S Y N_{f}=2 N_{c} Q C D$, arXiv:0909.3338 [hep-th].

[9] A. Mironov and A. Morozov, Proving AGT relations in the large-c limit, arXiv:0909.3531 [hep-th].

[10] G. Bonelli and A. Tanzini, Hitchin systems, $N=2$ gauge theories and $W$-gravity, arXiv:0909.4031 [hep-th].

[11] V. Alba and A. Morozov, Non-conformal limit of AGT relation from the 1-point torus conformal block, arXiv:0911.0363 [hep-th].

[12] Al. Zamolodchikov, Conformal Symmetry In Two-Dimensions: An Explicit Recurrence Formula For The Conformal Partial Wave Amplitude, Commun. Math. Phys. 96 (1984) 419.

[13] Al. Zamolodchikov, Two-dimensional conformal symmetry and critical four-spin correlation functions in the Ashkin-Teller model, Sov. Phys. JETP 63 (1986) 1061.

[14] Al. Zamolodchikov, Conformal symmetry in two-dimensional space: recursion representation of conformal block, Theor. Math. Phys. 73 (1987) 1088.

[15] L. Hadasz, Z. Jaskólski and P. Suchanek, Recursion representation of the Neveu-Schwarz superconformal block, JHEP 03 (2007) 032 [hep-th/0611266].

[16] V. A. Fateev, A. V. Litvinov, A. Neveu and E. Onofri, Differential equation for four-point correlation function in Liouville field theory and elliptic four-point conformal blocks, J. Phys. A 42 (2009) 304011 [arXiv:0902.1331 [hep-th]].

[17] A. Zamolodchikov, Higher equations of motion in Liouville field theory, Int. J. Mod. Phys. A 19S2 (2004) 510 [arXiv:hep-th/0312279]. 
[18] H. Sonoda, Sewing Conformal Field Theories. 2, Nucl. Phys. B 311 (1988) 417.

[19] A. B. Zamolodchikov and Al. Zamolodchikov, Structure constants and conformal bootstrap in Liouville field theory, Nucl. Phys. B 477, 577 (1996) [arXiv:hep-th/9506136]. 